\title{
The influence of the large scale mechanization of ware potato growing upon supply responsiveness in The Netherlands (1955-1978)
}

\author{
F.A.J. VAN DEN BOSCH \\ Eramus Univarsity, Rotterdam \\ C.A.P. VEERMAN \\ Graduate School of Management, Delft
}

\section{Summary}

This article brings together the findings of an investigation into the influence of large scale mechanization on the supply response of ware potatoes in The Netherlands (1955-1978).

For this purpose the considered period has been divided into two subperiods: the period before the introduction of the mechanization (1955-1965) and the period within which this mechanization started (1965-1978).

A theoretical supply response model has been developed. The estimation results of this model with respect to the two sub-periods indicate that mechanization sharply reduced the supply responsiveness. The results did not support the hypothesis that the gross margin should be preferred to the product price as an explanatory variable.

\section{Introduction}

In this paper we will present our findings from a study of the ware potato market between 1955 and 1978 . The principal question we will try to answer is whether and to what extent the mechanization of ware potato growing that has taken place during this period has significantly influenced the responsiveness of the acreage to changes in revenue from the crop.

For this purpose we have developed a simple supply response model in which the explanatory variables include the gross margins of the potato crop and of competing crops. In this connection, another question we have investigated is whether the acreage response can be better explained empirically by price or gross margin changes.

Potatoes are one of the last major crops without a regulated market in the EC. On the one hand this makes it possible to study the behaviour of the 
farmers under significantly fluctuating price conditions and on the other hand it is very important to know whether the price mechanism really works as it is believed to work.

In order to keep the subject of study as homogeneous as possible we restricted ourselves to the ware potato crop that is grown on one particular sort of soil: clay. ${ }^{1}$ Most of the ware potato crop is grown on this type of land and since The Netherlands is small there is no reason to suppose that differences of climate will have any significant effect on potato-growing, although clay ground is not concentrated in one particular geographical area. We have chosen the aggregate approach to investigate the responsiveness of the acreage to changes in prices.

In their article, Askari and Cummings (1977) give a survey of the research that has been undertaken using the Nerlove supply response model. We did not use this model, in spite of its impressive record, because of the following objections. Firstly, it is a one product model; other crops are not supposed to have any direct influence upon the decision about the particular crop. There have been a lot of attempts to solve this problem by deflating the price of the particular crop by means of the prices of the other crops. We will try to take prices of alternative crops directly into consideration, because of the possibility of farmers being inclined to think in absolute rather than in relative prices. Secondly, as Hill (1971) has pointed out, Nerlove's model assumes a constant technology. We will argue in the next section that during the period we are studying a sort of technological revolution has taken place in potato harvesting methods. In addition to this we will argue that gross margins are the relevant variables in the supply response equations and not prices.

Thirdly, there follows from Nerlove's model a specification for the estimator equation which contains the variable that is to be explained with a lag of one period. It is very difficult to substantiate the theoretical importance of this variable. Statistically speaking, the variable only serves as a means of depressing serial correlation. Therefore, we conclude there is room for an alternative way of specifying the estimator equation on the basis of a simple acreage decision model.

In Section 2 we describe the developments in the potato growing sector and analyse the structural changes that took place. In Section 3 our supply response model will be outlined and some comments will be made on the way the theoretical model has been estimated. The empirical results will be presented and discussed in Section 4. Section 5 contains our main findings and concluding remarks. 


\section{Developments in the structure of ware potato growing and sales}

In this section we will describe and explain the major developments in the production process. We have noted considerable technological changes in the production of this crop. We will divide the period under consideration into two sub-periods. Large scale mechanization did not begin until the second बff these periods. A very rapid growth in new sales openings (exports) occurrèd simultaneously with this development. We will explore the consequences off these changes in the production and sales structure below.

Table 1. Changes in the structure of ware potato growing (including se potatoes)

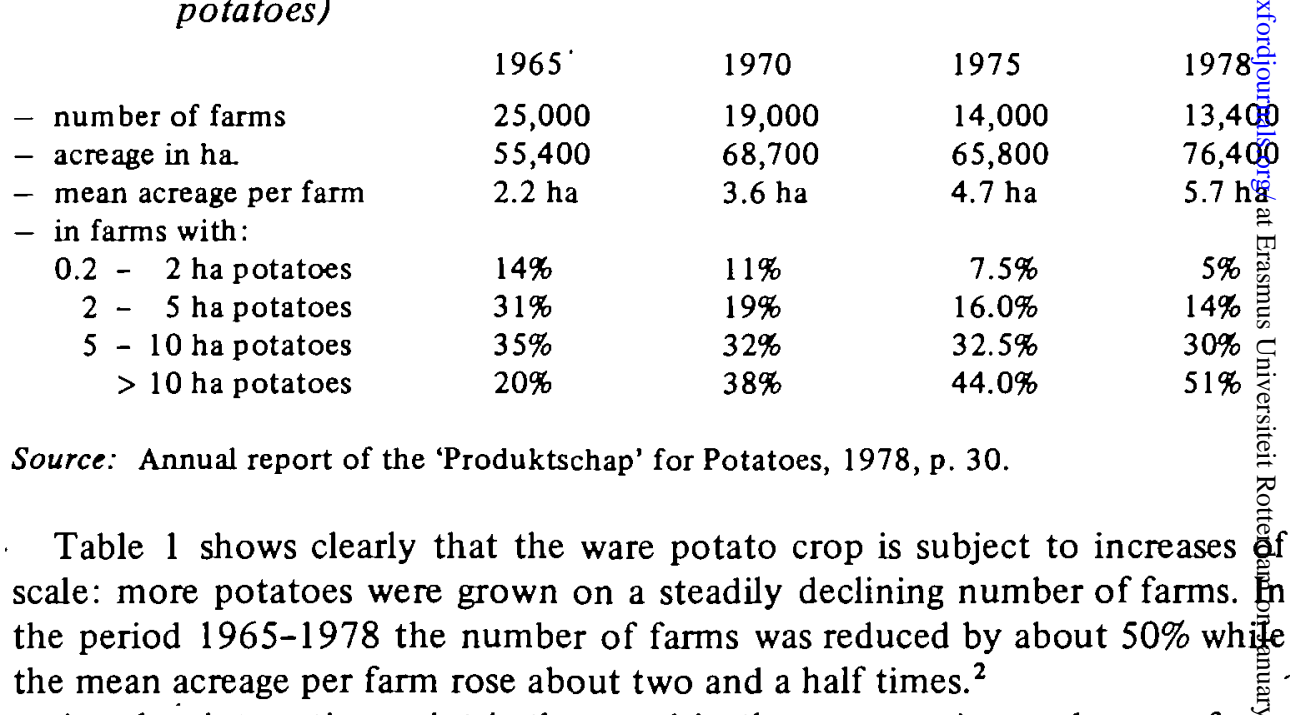

Another interesting point is the trend in the acreage. As can be seen from Figure 1, from 1955 to 1965 there was a slight downward trend and from 1965 onward a sharp upturn. This is a remarkable phenomenon and one might have expected technical factors to explain it, such as the decline in the area of agricultural land due to the building of houses, factories and roads, and changes in the regulations imposed on farmers by the Committee of Plant Pathology in order to prevent the spread of diseases caused by over-intensive cultivation. Both factors were in fact operative in the period under consideration, but in exactly the opposite direction to what one might expect. The area of agricultural land declined by about $25 \%$ from 1955 to 1978 and the regulations 
of the committee became considerably more tight: from one crop of potatoes every three years on a particular piece of land to one crop in four years.

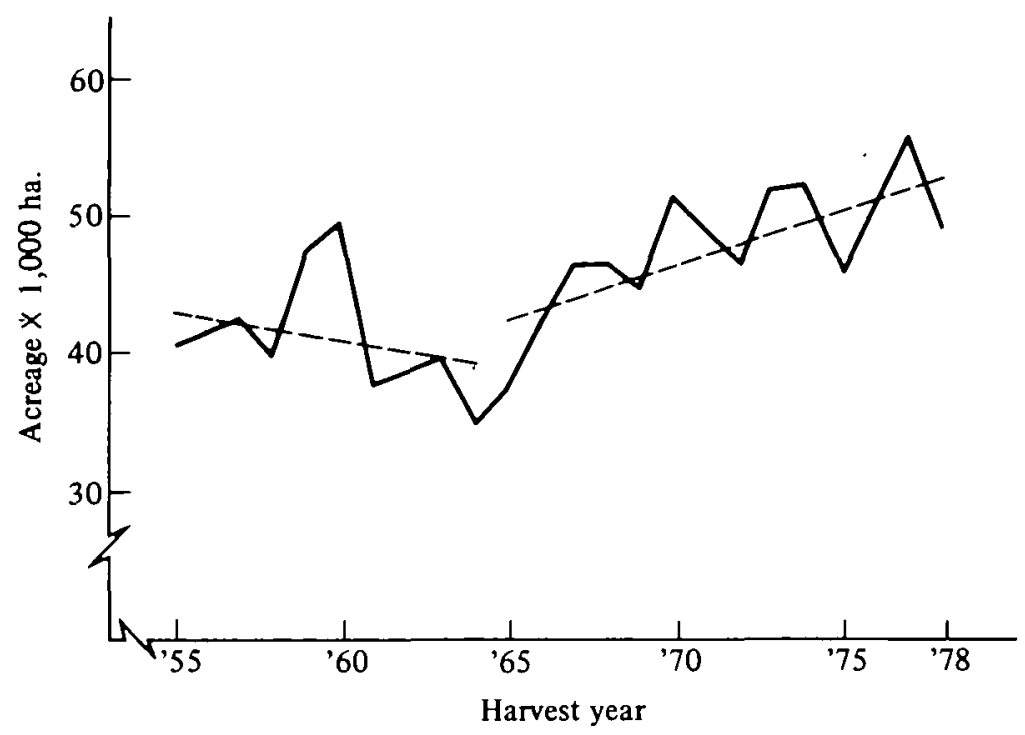

a The trend lines are estimated by simple linear regression.

Source: Agricultural Statistics of the Central Bureau of Statistics (C.B.S.) and Annual Reports of the General Dutch Plant Pathology Inspection Committee.

Figure 1. Acreage of ware potatoes grown on clay (excluding seed potatoes). ${ }^{\mathrm{a}}$

In our opinion, the explanation is to be found in changes in the production process during the sixties. After the War, potato-growing was still very labourintensive, especially as regards harvesting. By the end of the sixties the situation had changed fundamentally; the process had been mechanized practically throughout. Mechanization is not something which the individual farmer can introduce gradually, as it involves a chain of mutually dependent machines. If a farmer wanted to substitute capital for labour it had to be done rigorously. He had to invest not only in machines, but also in storage capacity, because the harvest period was drastically shortened and the traditional method of storage in heaps on the land now became too labour intensive and too dependent upon meteorological factors. ${ }^{2}$

Because we would like to compare the supply response with and without 
large scale mechanization, we have to divide the period into two sub-periods as mentioned above. We assume that the mechanization of potato growing started in the mid-sixties. However, it is very difficult to find adequate data as far as machines are concerned. This is due to the fact that in general the available data is not (or cannot be) crop specific. We assume that the mechanization of potato growing has led to the replacement of old machines with new types.

Table 2 illustrates the replacement process with respect to lifting machines. Columns (1) and (2) show numbers of labour intensive and labour extensive lifting machines. It appears that in 1965 the number of machines of the first type had decreased since 1960, while there had been a rapid increase in labour extensive machines. As Figure 1 shows, the introduction of mechanization coincided with the upturn in acreage.

Table 2. Numbers of labour intensive and labour extensive potato crop lifting machines $(1960=100)$.

\begin{tabular}{lll} 
Year & \multicolumn{2}{c}{ Number of lifting machines $(1960=100)$} \\
\hline & $\begin{array}{l}\text { Labour intensive } \\
\text { (without collection) }\end{array}$ & $\begin{array}{l}\text { Labour extensive } \\
\text { (with collection in bags, car etc.) }\end{array}$ \\
\cline { 2 - 3 } & $(1)$ & (2) \\
1960 & 100 & 100 \\
1965 & 62 & 168 \\
1970 & 51 & 199 \\
\hline
\end{tabular}

Source: Agricultural data, Agricultural Economic Institute (1976).

Another way of determining approximately in which years large scale mechanization began, is to look at the results of this process, namely the yields of the potato crop. In the period 1955-1964 the average yield was 30 tonnes per hectare and in the succeeding period (1965-1978) 36 tonnes per hectare. Moreover, the yield was higher than 30 tonnes per hectare every year in the second period.

The mechanization of potato growing has greatly influenced the flexibility of the supply response mechanism. The agricultural entrepreneur has freedom to alter the acreage put down to one crop or another. The other factor, the physical quantity of the produce, which in the short run determines the total supply of a crop, is subject to many factors such as the weather and disease.

In his efforts to achieve maximum profits, the farmer only has two instru- 
ments: minimizing production costs and enlarging the physical quantity. If we go into the first of the two more deeply (the second is of an entirely different character), and especially if we focus on the relative part of fixed costs in the total costs of the crop, it can easily be seen that this particular part has risen sharply because of the mechanization. ${ }^{4}$ The only way to depress the fixed costs per hectare is to enlarge the acreage under cultivation up to the point at which the available capacity of machinery or of storage is reached, or to the maximum acreage that can legally be planted. Potato growing demands far greater investment than other crops. So farmers are inclined to enlarge the acreage of potatoes because of the pressure of the high fixed costs.

This upward pressure on the acreage would surely have ceased very soon had it not been possible to export the consequent production surplus, which was still further enhanced by the rise in physical productivity per hectare due to technical improvements to the specific crop. ${ }^{5}$ However, an increase in exports created a new outlet for sales. To illustrate this development, exports (measured in tonnes) grew by $200 \%$ in the period 1963-1973. Another development with respect to the sales structure is the way the crop has been sold. This point is also important for studying price responsiveness. Although the percentage of the crop sold on the free market has declined during the period, more than $50 \%$ still finds its way to the customer through this channel.

\section{A simple model of the acreage planting decision}

In the previous section we dealt with the explanation of the trend line, now we will focus our attention on the fluctuations around the trend. As we stated before, the short run decisions with respect to production in agriculture are those concerning the acreage of the different crops that can be grown on the particular soil. Physical productivity per hectare is in the short run largely dependent on meteorological factors and in the long run can only be influenced to a limited extent by technological and chemical improvements to the crop. Thus farmers can only respond to changing market conditions by altering the acreages of the various crops.

Let us suppose that a farmer with a total farm acreage $a_{t}$ in the crop year $t$ is able to choose among $i(i=1 \ldots n)$ crops. We next assume that the costs of the respective crops consist of variable costs $V E_{i}$ and constant costs $C_{i}$, which are independent of the physical quantity produced per hectare. We define the expected gross revenue per hectare of culture $i\left(G R_{i}\right)$ as the product 
of expected physical quantity from a hectare in kilo's $\left(\mathrm{PQ}_{\mathrm{i}}\right)$ of culture $\mathrm{i}$ and the expected price of culture $i\left(P_{i}\right)$. Thus $G R_{i}=P Q_{i} \times P_{i}$. Finally we define the expected Gross Margin of culture i as: $G_{i}=G_{i}-V E_{i}$.

Now if we take the regulations of the Government with respect to the maximum acreage of potatoes fixed in order to prevent the spread of diseases we can formalize them as follows. Let $a_{t, i}$ be the maximum acreage of culture $i$ in crop year $t$; then $a_{t, i}<a_{t, i} \max <a_{t}$.

Profit maximization means logically the maximization of the sum of the gross margins of the respective crops. Ranking the gross margins of the respective crops according to their size logically means that the maximum acreage permitted must be of the crop with the largest gross margin, and then of the second largest, and so on.

Formally, if $\mathrm{j}$ is the crop with the highest GM:

$$
a_{t, j}=a_{t, j} \max . \text { but } a_{t, j} \max .<a_{t}
$$

If $a_{t}-a_{t, j}>0$ then the crop with the next highest GM,k is planted:

$$
\begin{aligned}
& a_{t, k}=a_{t, k} \max , \text { if } a_{t}-a_{t, j}>a_{t, k} \max . \\
& a_{t, k}=a_{t}-a_{t, j}, \text { if } a_{t}-a_{t, j}<a_{t, k} \max .
\end{aligned}
$$

This goes on until:

$$
a_{t}-\sum_{i=1}^{n} a_{t, i}=0
$$

This means that:

$$
\begin{aligned}
& a_{t, i}=f\left(G_{t, i}, \ldots G_{t, \mathfrak{i}} \ldots G \ldots M_{t, n}, a_{t, 1} \max . \ldots\right. \\
& \left.\ldots a_{t, i} \max . \ldots a_{t, n} \max . a_{t}\right)
\end{aligned}
$$

We assume that the partial differential quotients have the following values: ${ }^{6}$

$$
\frac{\delta \mathrm{a}_{\mathrm{t}, \mathrm{i}}}{\delta \mathrm{GM}_{\mathrm{t}, \mathrm{i}}}>0 ; \frac{\delta \mathrm{a}_{\mathrm{t}, \mathrm{i}}}{\delta \mathrm{GM}_{\mathrm{t}, \mathrm{j}}}<0 ; \frac{\delta \mathrm{a}_{\mathrm{t}, \mathrm{i}}}{\delta \mathrm{a}_{\mathrm{t}, \mathrm{i} \max .}}>0 ; \frac{\delta \mathrm{a}_{\mathrm{t}, \mathrm{i}}}{\delta \mathrm{a}_{\mathrm{t}}}>0
$$

with: $\mathrm{j}=1-\ldots \mathrm{n} ; \mathrm{j} \neq \mathrm{i}$. 
The basic model as described by eq. (1) contains gross margins rather than prices as in many other models. In principle, it seems that the gross margin should be preferred to the price in supply response equations, especially if the technology cannot be regarded as constant. For by definition, the gross margin contains both the price and other relevant information: the results of the mechanization (the yields) and the consequences for the expenditures which are relevant in the short run (the variable expenditures).

In principle the supply response has to be estimated by means of a simultaneous model. Such a model can be specified in the case of $n$ crops by deriving ( $n-1)$ equations in accordance with eq. (1) and the legal acreage restrictions. However, if it can be demonstrated that there are no significant competing crops to the potato for the same soil, it is reasonable to estimate the supply response by means of a single equation. We will return to this point.

In trying to specify an estimator equation from eq. (2), we have to face a number of problems. First there is the problem that the economic variables in eq. (1) represent expectations. We have chosen the simple hypothesis that expectations are based upon the actual values for the previous two years, with equal weight being attributed to each of them. The second problem is the availability of data on the gross margins. There are no separate data on the gross margins of the different crops. This problem can be solved in two ways. There is the possibility of substituting the product prices as proxies for the GM's. We have adopted this solution in our estimation procedures.

To be more specific, we have considered two product prices for potatoes, one that represents the average product price over the whole harvest year from August until June (PPY) and one that represents the average product price over a shorter period from August till February (PPJ). The choice of this variable may be defended by the argument that most farmers make the planting decision no later than January, by which time most of them have already bought their seed potatoes. By the end of January most of the potato crop has been sold (nearly 70\%). Other crops, such as wheat, are sown even earlier - in the autumn.

The other way to overcome the difficulty of lack of data is to estimate the GM of potatoes separately. Whereas we did all our estimations of the variables in annual percentage changes ${ }^{7}$ then proceeded as follows (let the tilde above every variable denote the percentage change). The equation is true for every crop: ${ }^{8}$ 


$$
\tilde{\mathrm{GM}} \mathrm{t}=\frac{\Delta \mathrm{GM}_{\mathrm{t}}}{\mathrm{GM}_{\mathrm{t}-1}} \text { and } \mathrm{GM}_{\mathrm{t}}=\mathrm{PQ}_{\mathrm{t}} \cdot \mathrm{P}_{\mathrm{t}}-\mathrm{VE}_{\mathrm{t}}
$$

so:

$$
\bar{G}_{\mathrm{M}}=\overline{\mathrm{P}} \bar{Q}_{\mathrm{t}} \cdot \lambda_{\mathrm{o}}+\tilde{\mathrm{P}}_{\mathrm{t}} \cdot \lambda_{\mathrm{o}}-\overline{\mathrm{V}} \mathrm{E}_{\mathrm{t}} \cdot \lambda_{1}
$$

To solve the problems caused by lack of data on variable costs we undertook the following division of these costs into two parts:

$$
\mathrm{VE}_{\mathrm{t}}=a_{\mathrm{t}} \cdot \mathrm{PGP}_{\mathrm{t}-1}+\mathrm{R}_{\mathrm{t}}
$$

where: $\quad a_{\mathrm{t}}=$ quantity of seed potatoes in tonnes per hectare.

$\mathrm{PGP}_{\mathrm{t}-1}=$ price of seed potatoes per tonne from the previous crop year. $R_{t}=$ rest of the variable costs.

The following remarks should be borne in mind. First, the cost of seed potatoes is the largest cost item (nearly 70\%). Second, farmers buy their seed potatoes from the previous seed potato crop. When we transform this equation into percentage changes ${ }^{9}$ we get:

$$
\overline{\mathrm{V}} \tilde{E}_{\mathrm{t}}=\lambda_{2} \cdot \tilde{\mathrm{P}} \overline{\mathrm{G}} \tilde{\mathrm{P}}_{\mathrm{t}-1}+\lambda_{2} \cdot \tilde{a}_{\mathrm{t}}+\lambda_{3} \cdot \tilde{\mathrm{R}}_{\mathrm{t}}
$$

If, after we have determined the values of $\lambda_{0}, \lambda_{1}, \lambda_{2}$, and $\lambda_{3}$, we substitute this equation into (2) and suppose $a_{t}=0$, we get the estimator equation. We have calculated the average values of $\lambda_{0}$ and $\lambda_{2}$ on the basis of the available data for the period 1971-1976, in the most important regional production areas (the values of $\lambda_{1}$ and $\lambda_{3}$ ) follow directly from the conditions stated in Notes 3 and 4 to the present section.

As above calculations for the potato crop reveal, it is very difficult to take all the GM's of the different crops into consideration in trying to explain supply response in the potato crop.

In order to get an impression of the relevance of the other crops for potato supply response, we estimated the influence of gross revenue from the main competing crops. Cereals (especially wheat) and sugarbeet are the chief alternative crops in the period under consideration. The following equation gives an estimate of the influence of these two main competing crops for the first of the two sub-periods (1955-1964). We necessarily anticipate the empirical results of our basic model presented in Section 4. 


$$
\begin{aligned}
& a_{\mathrm{t}}=0.50 \tilde{\mathrm{P}} \tilde{\mathrm{P}} \tilde{\mathrm{J}}_{\mathrm{t}-1}+0.22 \tilde{\mathrm{P}} \tilde{\mathrm{P}} \tilde{\mathrm{t}}_{\mathrm{t}-2}-0.13 \mathrm{GRS}_{\mathrm{t}-1}-0.15 \mathrm{GRW}_{\mathrm{t}-1}-2.97 \\
& \begin{array}{llll}
(7.39) \quad(2.23) \quad(-1.50) \quad(-1.22) \quad(-1.99) & 0
\end{array} \\
& \mathrm{R}^{2}=0.85 ; \mathrm{D} \cdot \mathrm{W} .=2.34
\end{aligned}
$$

It appears that gross revenues from sugarbeet (GRS) and wheat (GRW) do not have a statistically significant coefficient, although the signs are according to our assumptions (see also the list of symbols under Table 4). We also tried other modifications of our basic model, but the influence of the competing crops did not appear significant in any of the estimates with respect to the first or second sub-periods.

We also tried to corroborate this finding in another way, namely by comparing the development of prices paid to the farmer with the yields for the three main crops: ware potatoes, sugarbeet and wheat. Table 3 presents this comparison for the two sub-periods. First it appears that the average price and the yield of the potato crop have the highest percentage change, $60 \%$ and $20 \%$ respectively. Second, it appears that the gross revenue ${ }^{10}$ of the potato crop is far greater than that of the other crops, although according to the values of the variation coefficients ${ }^{11}$ there is more uncertainty about the expected revenue.

Table 3. Comparison of the average farm gate prices, the average yields and the variation in both variables for three main crops.

\begin{tabular}{lcllll}
$\begin{array}{l}\text { main } \\
\text { competing } \\
\text { crops }\end{array}$ & sub-period & $\begin{array}{l}\text { average } \\
\text { farm gate price } \\
\text { (guilders/ton) }\end{array}$ & $\begin{array}{l}\text { coefficient } \\
\text { of } \\
\text { variation }\end{array}$ & $\begin{array}{l}\text { average } \\
\text { yield } \\
\text { (ton/hectare) }\end{array}$ & $\begin{array}{l}\text { coefficient } \\
\text { of } \\
\text { variation }\end{array}$ \\
\hline ware & $1955-1964$ & 129 & $15 \%$ & 30 & $8 \%$ \\
potatoes & $1965-1978$ & 209 & $63 \%$ & 36 & $8 \%$ \\
sugarbeets & $1955-1964$ & 53 & $17 \%$ & 43 & $13 \%$ \\
& $1965-1978$ & 74 & $17 \%$ & 46 & $7 \%$ \\
wheat & $1955-1964$ & 298 & $10 \%$ & 4.4 & $11 \%$ \\
& $1965-1978$ & 395 & $12 \%$ & 5.2 & $13 \%$
\end{tabular}

As for the second group of variables in eq. (1), it is interesting to note that for the main crop area in The Netherlands the legally permitted acreage for potatoes, $25 \%$ of the cultivable land, is not reached yet. Over the period 
1965-1976 the percentage of potatoes in the total area of claysoil in the South-West area did not exceed $19 \% .^{12}$

Apart from the economic factors involved in the acreage decision, there are psychological and technical ones. Ingersent (1969) and Revell (1974) found a significant value for a dummy variable representing the amount of rainfall in the previous autumn. We have not been successful in trying to prove such a variable significant in our country, neither were we able to prove Tomek's (1972) suggestion of a significant mechanisation dummy variable.

\section{Empirical results}

According to the analysis in Section 2 and 3 the supply response mechanism has been estimated separately for the first sub-period (1955-1964) before the large scale mechanization actually started, and for the second sub-period (1965-1978) in which mechanization did take place. In doing so, we are able to estimate the influence of mechanization on the supply responsiveness.

The basic equation (1) has been estimated in annual percentage changes, and we left out the possible competing crops and acreage restriction as was explained above. We took two variants of the farm gate price: one as the average of part of the year and the other one as the average of the whole harvest year, each with a time lag of one and two years. The results are presented in Table 4.

It is clear that the results of the model specification that are estimated are in accordance with the parameter restrictions as specified in the theoretical model. The signs of the variables all correspond to the theoretical expectations. It is a remarkable fact that the results, statistically speaking, are better for the first period than they are for the second. It is also interesting to note the statistically more adequate results of the price variables in relation to the contructed GM variables; where the average pre-February price PPJ does slightly better than PPY.

Furthermore, the suggestion that the memories of the farmers may be short as manifested by the decreasing relevance of the price variable, which lagged two years from the first to the second period, is an interesting one. Finally a considerable decline in the relevance of the price variable as well as the GM variables may be concluded from these estimations, which is possibly the empirical support for the points we made in Section 2 when we discussed the increase in mechanization and the subsequent upward pressure on the acreage 
of the potato crop combined with a decline in the flexibility to respond to changed market conditions.

Table 4. A number of model specifications explaining the percentage change of the acreage of ware potatoes (two periods: 1955-1964 and 1965-1978; the Netherlands).

explanatory

variables sub $G \tilde{M}_{t-1} \quad G \bar{M}_{t-2} \quad P P Y_{t-1} P P Y_{t-2} \quad P P J_{t-1} \quad P P J_{t-2} \quad$ Constant $R^{2} \quad$ D.W periods

1955-1964

specification

\begin{tabular}{|c|c|c|c|c|c|c|c|}
\hline $\begin{array}{l}+0.22 \\
(2.86)\end{array}$ & $\begin{array}{l}+0.24 \\
(2.93)\end{array}$ & & & & & $\begin{array}{c}-5.25 \\
(-1.87)\end{array}$ & 0.47 \\
\hline$\div$ & & $\begin{array}{l}\overline{+0} . \overline{35} \\
(3.48)\end{array}$ & $\begin{array}{l}\overline{+0} . \overline{31} \\
(3.10)\end{array}$ & & & $\begin{array}{l}-5 . \overrightarrow{46} \\
(-2.08)\end{array}$ & $0.5 \overline{4}$ \\
\hline 3 & & & & $\begin{array}{l}+0.46 \\
(5.78)\end{array}$ & $\begin{array}{l}+0.21 \\
(2.62)\end{array}$ & $\begin{array}{l}-4.02 \\
(-2.33)\end{array}$ & 0.78 \\
\hline
\end{tabular}

$1965-1978$

specification

$$
\begin{aligned}
& 1+0.09+0.06 \\
& \begin{array}{llll}
+0.09+0.06 & -1.27 & 0.59 & 0.91
\end{array} \\
& \underline{(4.20)} \stackrel{(2.80)}{+0.09} \overline{+0} . \overline{06}----\frac{(-0.67)}{-0.88}--- \\
& 3 \\
& ---\underline{(3.70)} \underline{(2.38)} \\
& \overline{+0} . \overline{2} \overline{+0.04} \frac{(-0.44)}{-0.07} \frac{0.53}{0.55} \frac{0.91}{0.86} \\
& \text { (4.26) (1.24) (-0.04) }
\end{aligned}
$$

\section{List of symbols:}

- : annual percentage change of the variable;

$\mathrm{t}-1$ : time lag, measured in harvest years;

GM : gross margin of ware potatoes;

PPY : farm gate price of ware potatoes, average of the whole harvest year;

PPJ : farm gate price of ware potatoes, average of a part of the harvest year (Aug.-Jan.);

$\mathrm{R}^{2} \quad$ : corrected squared correlation coefficient;

D.W : Durbin-Watson statistic;

(...) : between parentheses the t-ratios. 


\section{Concluding remarks}

In this study an attempt has been made to investigate the influence of large scale mechanization upon the supply response of ware potatoes. The basic idea is that a still continuing mechanization of this crop, meaning rapidly growing constant costs, leads first to decreasing supply responsiveness and second to an upward pressure on the supply (acreage). To investigate this, the considered period was divided into two periods, namely the period before and the period within which the mechanization started. Several indicators were developed to justify this division.

A simple theoretical supply response model has been presented, that differs at several points from the traditional approach. The model contains the gross margin of the crop concerned and those of the competing crops. Besides all that, the model shows the influence of acreage restrictions upon the supply response.

On several grounds it seemed plausible to us that there are no significant substitutes for the potato crop. This finding justifies the use of a single product equation. The estimation results of the model with respect to the two subperiods lead to the conclusion that mechanization of the potato crop sharply reduced the supply responsiveness. The price elasticity and the elasticity of the gross margin with respect to acreage were reduced on average to one fourth and to half of the values in the period before mechanization. We did not find evidence to support the conclusion that the gross margin should be preferred to the product price as an explanatory variable. Our empirical results indicate decreasing supply responsiveness. An important consequence of this development is the reduced possibility of the supply to adapt to changing economic conditions.

\section{NOTES}

1. Ware potatoes grow also on sandy soils. In 1978 the average acreage per farm was less than a third of that of farms on clay soils.

2. The mean acreage per farm amounted to 1,1 hectares in 1959 and 1,3 hectares in 1963.

3. The average capacity of potato storages per hectare of potato crops increased by $75 \%$ in the period 1963-1968.

4. The yearly average growth rate of the fixed costs per unit of acreage was $3 \%$ in the period 1963-1965, 8\% in the period 1966-1970 and 10\% in the period 1971-1976. These figures relate to farms in a major production region. 
5. The average gross production in tonnes per hectare rose from 28.2 in 1955-1959 to 30 in 1960-1964 and had reached 34 tonnes per hectare in the period 1965-1969.

6. The partial differential quotients can be equal to zero if, in spite of a rise in the gross margin, the acreage cannot further increase because of restrictions caused by the availability of other factors of production.

7. The estimation of the model with the variables transformed into changes gives the well known econometric advantages - for example, the elimination of multicollinearity of the regressors because of an underlying trend in the variable. Besides this, the estimated model yields values of the respective elasticities directly.

8. $\lambda_{0}=\frac{\mathrm{PQ}_{\mathrm{t}-1} \cdot \mathrm{P}_{\mathrm{t}-1}}{\mathrm{GM}_{\mathrm{t}-1}} ; \lambda_{\mathrm{l}}=\frac{\mathrm{VE}_{\mathrm{t}-1}}{\mathrm{GM}_{\mathrm{t}-1}} ; \Delta \mathrm{P}_{\mathrm{t}} \cdot \Delta \mathrm{PQ}_{\mathrm{t}}=0$ and $\lambda_{0}-\lambda_{1}=1$.

9. $\lambda_{2}=\frac{a_{t-1} \cdot P^{P G P} t-1}{V E_{t-1}} ; \lambda_{3}=\frac{R_{t-1}}{V E_{t-1}} ; \Delta a_{t} \cdot \Delta \mathrm{PGP}_{t}=0$ and $\lambda_{2}+\lambda_{3}=1$.

10. A comparison with respect to the contribution margins would be preferable.

11. The low value of the variation coefficient of the farmer's price of wheat and sugarbeet could be explained by the fact that both are regulated crops.

12. This percentage still grows and reached $21,5 \%$ in the province of South-Holland in 1978. This development also supports the findings with respect to the competing crops of the potato crop.

\section{REFERENCES}

Askari, H. and Cummings, J.T. (1977). Estimating agricultural supply response with the Nerlove model: A survey. International Economic Review, pp. 257-292.

Hill, B.E. (1971). Supply response in crop and live stock production. Journal of Agricultural Economics, pp. 287-293.

Ingersent, K.A. (1969). Models, to explain annual changes of the potato acreage in Great Britain since 1955. The Farm Economist, pp. 428-443.

Revell, B.J. (1974). A regional approach to the potato acreage planting decision. Journal of Agricultural Economics, pp. 55-64.

Tomek, W.G. (1972). Distributed lag models in cotton acreage response: A further result. American Journal of Agricultural Economics, pp. 108-110.

C.P. Veerman and F.A.J. van den Bosch

Interunive rsitaire Interfaculteit

Bedrijfskunde-Delft

Poortweg 6-8

Delft

The Netherlands 